d'hydrocarbures liquides et devant les réserves relativement faibles en produits naturels de toute la planète, ne va-l-on pas voir la houille blanche prêter son concours à la houille noire pour obtenir artificiellement ces hydrocarbures liquides. Toutes ces sources d'énergie ne seront donc plus des concurrentes comme on voulait le dire, mais bel et bien des collaboratrices.

En effet, déjà de nombreux pays ont abordé le problèmo de l'obtention des carburants en partant des hydrocarbures solides (houilles, lignites, pyroschistes, tourbes, etc.) ; les uns le font dans le but de conserver leur indépendance; les autres pour avoir des produits moins coûteux ; d'autres, enfin, en prévision de l'épuisement des sources naturelles de pétrole.

Ne voit-on pas les Etats-Unis d'Amérique, pays du naphte cependant, envisager l'utilisation prochaine de leurs immenses réserves de schistes bitumeux, déjà évaluées à 108 milliards de lbarils - soit 17 milliards de tonnes de pétrole brut. - C'est ce qui ressort nettement de la dernière assemblée générale annuelle de I'Américan Petroleum Institute.

En France, le problème de l'alimentation en carburants se pose plus aigu que dans beaucoup d'autres pays, vu la pauvreté à peu près certaine du sous-sol et la consommation qui croît constamment. Pour l'an dernier, nos besoins ont dépassé 6 millions et demi de tonnes de produits pétrolifères et notre production s'en est tenue à 300.000 tonnes environ, en y comprennant Péchelbronn, Autun, Gabian, les benzols et les alcools.

On sait que les Allemands, placés dans une situation identique à la nòtre, ont résolu la question par l'obtention de carburants synthétiques à partir des combustibles solides ef principalement des lignites. A la suite de recherches, nuxquelles nombre de savants et inventeurs français se sont intéressés, on a reconnu que lie seul moyen réellement industriel pour arriver aux carburants de synthèse à un prix convenable, résidait dans l'hydrogénation des huiles brutes provenant de carbonisation de houilles et surtout des lignites et des pyroschistes.

L'opération comprend donc deux phases successives, mais bien distinctes l'une de l'autre ; premièrement, la carbonisation de la roche hydrocarburée - on désigne improprement cette phase sous le nom de distillation - et, deuxièmement, l'addition, sous conditions déterminées, d'hydrogène aux huiles ainsi produites, pour arriver le plus près possible de la saturation.

La première phase s'effectue par les propres moyens de la substance mise en œuvre; une partie des calories qu'elle renferme étant employés à la transformation du restant : mais, dans la seconde, il faut obligatoirement un apport d'hydrogène et c'est là où nous envisageons le concours de la houille blanche.

Jusqu'à maintenant, le coût élevé de l'hydrogène a été considéré comme le plus grand obstacle ì l'obtention pratique des carburants de synthèse en partant des combustibles solides. On évalue, dans les uas les plus propices, à 900 mètres cubes, la quantité d'oxyggène utile à la production d'une tonne d'essence. Quand on hydrogène directement le charbon, il en faut 2 à 3 fois plus et c'est pour cela qu'on abandonne presque complètement ce mode de traitement, on se rend compte aussitòt, en appliquant à l'hydrogène le prix actuel de 25 à 30 centimes le mètre cube, de combien il grève le coût de l'essence finale. Il y a donc nécessité, en première ligne, d'abaisser le prix de revient du gaz hydrogène. C'est là que chez nous, la houille blanche a un rôle tout indiqué.

Les moyens d'obtenir l'hỵdrogène sont nombreux, nous n'en voulons pas faire l'énumération ; ceux qui pc'uvent frecevoir des applinations industrielles exigent des consommations élevés d'énergie qu'on ne peut songer à demander' qu'à la houille blanche.

La région en France qui est privilégiée pour renfermer des lignites extrêmement propres à la fabrication des carburants, correspond sensiblement an bassin idu Rhône ; c'est là que gisent les lignites de Fuveau, de Manosque, de Bagnols-surCèze, de la Tour-du-Pin, de Soblay, etc., etć. (on pourrait y ajouter des pyroschistes de Vagnas, de Boson, des Causses, du Jura, etc.) dont la plupart sont inutilisés aujourd'hui. Or, on parle de plus en plus de l'aménagement du Rhône au double point de vue navigation et force motrice ; la logique exige que celle-ci soit utiliséc à mettre en valeur les ressources régionales, dont les lignites, comme nous venons de le dire, font partie.

Nous savons, d'autre part, que le ministre des Travaux publics vient de mettre à l'enquête le projel du grand barrage de Génissiat (Ain). On se sonvient que ce projet, qui date de près de 30 ans, comporte dans les Gorges du Rhône, entre Seyssel et Bellegarde, un barrage de 80 mètres de hauteur environ qui transformera la valléc en un lac de 23 kilomètres de longueur, quoique ne couvrant que 380 hectares. Il en résultera non seulement la navigation possible entro Genissiat et P'ougny, près de Genève, mais également une source d'énergie évaluée à 59.000 Kilowatts au moment de l'étiage et à 200.000 et plus lors des hautes eaux.

Ce premier aménagement du Rhône peut donc être le point. de départ de l'hydrogène bon marché et par suite de l'obtenlion à bon compte des carblurants synthétiques, qui, cux, seront cent pour cent nationaux, puisqu'ils proviendront des lignites que nous avons méprisés à peu près totalement jusqu'aujourd'hui.

La houille blanche a permis il y a cinquante ans, à l'aluminium de devenir un métal d'usage courant, elle permettra demain à nos inépuisables réserves de roches pétroligènes de se transformer pratiquement en essence.

\title{
Expériences sur les piles de barrages déversoirs à profil aérodynamique et à contractions nulles
}

Note ${ }^{(1)}$ de MM. Léopold Escande et Georges Sabathé.

L'un de nous $\left(^{2}\right)$ avait étudié le profil optimum de barrage déversoir dont il avait indiqué la détermination pratique. Dans ce même travail, il avait proposé une méthode pour la détermination de piles à profil aérodynamique susceptibles de présenter un net avantagé vis-à-vis des profils usuels.
La méthode est la suivante : on trace, dans un plan, un profil d'aile biconvexe symétrique ; on déforme ensuite ce plan, en l'appliquant sur la surface cylindrique constiluanl le déversoir, de telle sorte que le profil, d'axe normal aux génératrices du barrage, ait son maître couple au voisinage 
du point le plus haut du sommet. On nbtient ainsi, sur le barage lui-mêne, wne couble qui conslitue la base de la pile: la surface lalérale de celle-ci esl alors délerminée par l'ensem-
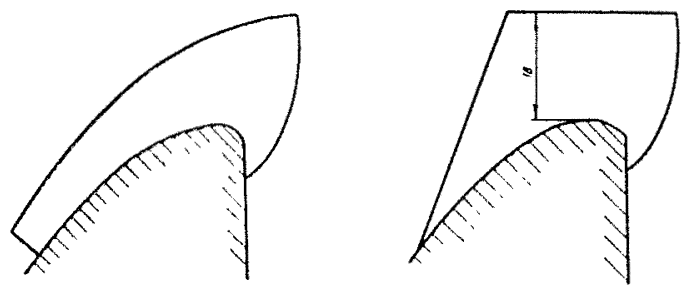

Pile aerodynamique

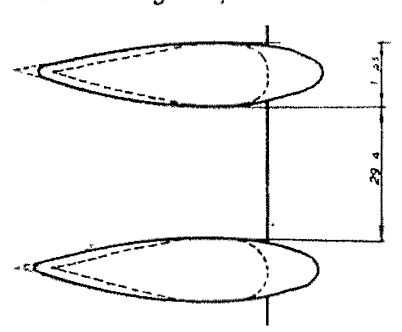

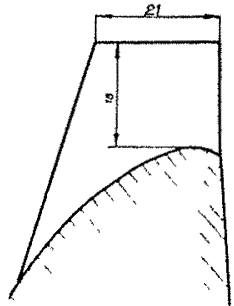

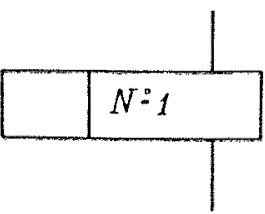

Fig. 1 tance d'axe en axe entre piles est de $0^{\mathrm{m}}, 294$; l'extrème pointe, du profil est tronquée ( $i g .1)$.

l'écoulement cst extrêmement régulier (fig. 2) ; après son passage au droit du maître couple la nappe déversante ne décolle pas de la pile el s'épanouit bur le parement aval du barrage ; les contraclions ne sont pas visibles.

Exprimons le débit du déservoir par la formule

$$
\mathrm{Q}=m\left[\mathrm{~L}-\frac{n \alpha h}{10}\right] h \sqrt{2} \mathrm{gh},
$$

dans laquclle $m$ est le coefficient de débit du déservoir en fabsence de piles, $n$ le nombre de contractions, $L$ la longueur forìle du débouché du seuil entres piles, $h$ la charge au désus du seuil et $\alpha$ un coefficient caractérisant la contraction.

On trouve, pour $h=0^{\mathrm{m}}, 16, \mathrm{~L}=1^{\mathrm{m}}, 469 \mathrm{el} n=10$, que $a$ a unc valeur sensiblement nulle.

En supprimant toute la portion de piles aérodynamiques siluée à l'aval du maître couple, nous nous sommes rendı comple que ccite suppression ne modifie en rien la valeur du débit vis-à-vịs du cas des piles entières et ne trouble en lien la régularité de l'écoulement (fig. 33).

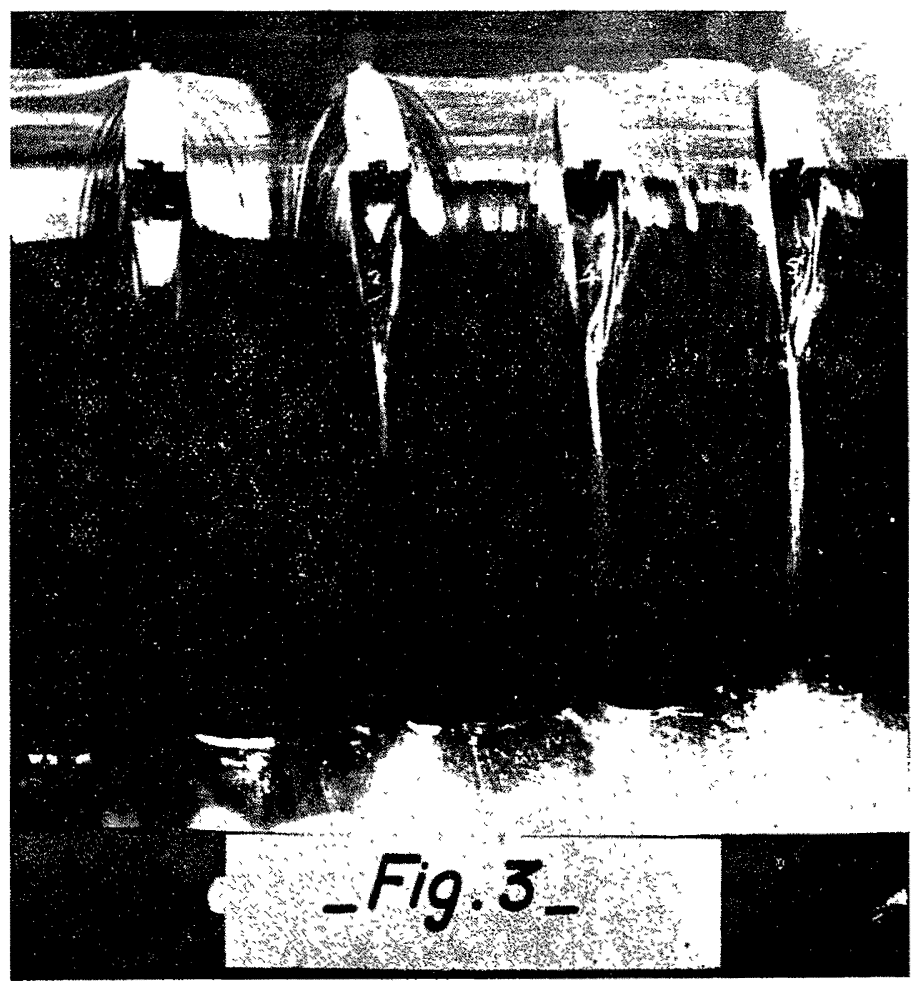

Nous avons enfin comparé les résultats ainsi obtenus à ceux que fournissent trois autres profils de piles, montés sur le même barrage et dont la figure 1 donne les caractézistiques ; les contractions deviennent visibles et l'on obtien! avec la même charge de $0^{\mathrm{m}}, 16$ au-dess'ıs du seuil, les valeurs suivantes de $d$ :

Poiur le profil $1 \ldots \ldots \ldots \ldots \ldots=0,10$

Poux le profil $2 \ldots \ldots \ldots \ldots \ldots=0,17$

Pour le profil $3 \ldots \ldots \ldots \ldots \ldots=0,70$

Cies résullats montrent le net avanlage des piles à profil aérodynamique.
(1) Séance du 30 mars 1936.

(2) L. Escande Revue Science el Indusirie, 236, septembre et octobre 1933, p. $\$ 67$, seuil el il est surmonté de piles déduites d'un profil Joukowsky de longueur $0^{\mathrm{m}}, 50$ de maîlre couple $0^{\mathrm{m}}, 1065$. La dis- 